\title{
Study progression of anti-angiogenetic therapy and its combination with other agents for the treatment of advanced hepatocellular carcinoma
}

\author{
Xinlei Gong, Shukui Qin \\ Department of Medical Oncology, PLA Cancer Center of Bayi Hospital Affiliated to Nanjing University of Chinese Medicine, Nanjing 210002, \\ China \\ Contributions: (I) Conception and design: S Qin; (II) Administrative support: S Qin; (III) Provision of study materials or patients: All authors; (IV) \\ Collection and assembly of data: All authors; (V) Data analysis and interpretation: All authors; (VI) Manuscript writing: All authors; (VII) Final \\ approval of manuscript: All authors. \\ Correspondence to: Shukui Qin, MD. Department of Medical Oncology, PLA Cancer Center of Bayi Hospital Affiliated to Nanjing University of \\ Chinese Medicine, Nanjing 210002, China. Email: qinsk@csco.org.cn.
}

\begin{abstract}
Hepatocellular carcinoma (HCC) is a common malignancy worldwide and is especially prevalent in the Chinese population. Treatment of advanced HCC has posed a considerable challenge to clinicians and highly effective therapies remain elusive. Angiogenesis contributes greatly to the pathogenesis, invasion and metastasis of HCC. Therefore, anti-angiogenesis therapy could be instrumental to the treatment of HCC. In recent years, several anti-angiogenesis drugs have generated significant outcomes in a few key clinical studies, and in this light, anti-angiogenesis therapy has become a critical aspect of comprehensive treatment of HCC. In this article, to provide a reference for clinicians, we review these advances and discuss the future direction of development.
\end{abstract}

Keywords: Hepatocellular carcinoma (HCC); anti-angiogenesis; treatment; drugs; research progress

Submitted Jun 16, 2018. Accepted for publication Oct 31, 2018.

doi: $10.21037 / \mathrm{hbsn} .2018 .11 .04$

View this article at: http://dx.doi.org/10.21037/hbsn.2018.11.04

Primary liver cancer (PLC) is one of the most common malignant tumors in the world, and hepatocellular carcinoma (HCC) accounts for about $90 \%$ of the cases. There were 841,000 newly diagnosed cases and more than 782,000 related deaths recorded worldwide, making HCC the fourth most common cause of cancer-related deaths globally, with China alone accounting for about $50 \%$ of the total number of cases and deaths (1). Additionally, the incidence rate and mortality rate rank fourth and second respectively, among all malignancies in China (2). According to the statistical results of the Global Burden of Disease Liver Cancer Collaboration study (3), between 1990 and 2015, liver cancer incident cases increased by $75 \%$. It is estimated that there were 854,000 incident cases of liver cancer and 810,000 deaths globally in 2015, with the incident cases and deaths in China being 466,000 and

\section{2,000 respectively.}

The prognosis of the advanced HCC, including Barcelona [Barcelona Clinic Liver Cancer (BCLC)], stage $\mathrm{C}$ and $\mathrm{D}$ types, is very poor. Even with the best supportive care (BSC) for these patients, the median survival period extends only from 6 to 9 months in European and American patients, and from 3 to 4 months in Asian patients (except for Japan). Because of its insidious onset, aggressive invasion, rapid progression and the existence of initial liver disease (hepatitis, liver cirrhosis), the treatment of advanced HCC is also very difficult, and there are no highly effective therapies yet available.

HCC is one of the typical vascular-rich tumors. The complex vasculature and its rich branches within liver cancer facilitate cancer-cell-produced angiogenesis promoting factors to promote the invasion and metastasis of 
cancer. Previous research and data have shown that vascular endothelial growth factor (VEGF) and VEGF receptor (VEGFR)-2-mediated angiogenesis contribute greatly to HCC pathogenesis. Therefore, the understanding of tumor angiogenesis is an important target for research in the treatment of liver cancer, and could be of great practicability as an effective therapy.

Sorafenib is an oral multi-kinase inhibitor with dual antitumor effects. For one, sorafenib can directly inhibit the growth of tumor cells by interfering with the RAF/MEK/ ERK signaling pathway. Additionally, it can also block the tumor angiogenesis process through repressing VEGFR and platelet derived growth factor receptor (PDGFR). At present, sorafenib is the only evidence-based systemic treatment option for patients with advanced HCC. It has already been approved in more than 180 countries and regions for the treatment of inoperable or metastatic HCC.

Since sorafenib was approved for HCC almost 11 years ago, a series of trials assessing molecular targeted agents, such as brivanib (4,5), sunitinib (6), linifanib (7), ramucirumab (8), everolimus (9), tivantinib (10) and axitinib (11), etc., have failed to improve upon outcomes achieved by sorafenib in the first-line setting or compared with BSC in the second-line setting after sorafenib therapy. However, this situation has changed recently. Several new targeted drugs and immune checkpoint inhibitors have shown positive results in phase 3 trials, and major breakthroughs have been achieved in the treatment of advanced HCC. This review intends to briefly discuss some of these pivotally successful HCC-related trials.

\section{Regorafenib}

Regorafenib is an oral multi-targeted inhibitor of multiple kinases including VEGFR 1-3, TIE2, c-kit, RET, wild type or V600-mutated B-RAF, PDGFR and fibroblast growth factor receptor (FGFR). The difference between sorafenib and regorafenib is that a fluorine atom takes the place of a hydrogen atom on a benzene ring. Therefore, it has also been called "second-generation sorafenib". A multi-center, open-label, phase II study leaded by Bruix (12) assessed the safety and efficacy of regorafenib in patients with advanced HCC that had progressed following sorafenib treatment. The results were promising and the safety profile was acceptable.

On this basis, a phase III study (RESORCE, NCT01774344) was conducted. This randomized, doubleblind, parallel-group, phase 3 trial (13) was carried out at
152 sites in 21 countries globally. A total of 573 patients with HCC who progressed on sorafenib and had ChildPugh liver function class A were enrolled. All participants were randomly assigned to receive regorafenib treatment or placebo at a ratio of 2:1, and stratified by geographical region, Eastern Cooperative Oncology Group (ECOG) performance status, macrovascular invasion, extrahepatic disease, and $\alpha$-fetoprotein level. The primary endpoint was overall survival (OS). Ultimately, regorafenib improved OS with a hazard ratio of 0.63 (95\% CI: $0.50-0.79$, $\mathrm{P}<0.0001)$, and median OS (mOS) was 10.6 months $(95 \%$ CI: 9.1-12.1 months) for regorafenib versus 7.8 months (95\% CI: 6.3-8.8 months) for placebo. The improvement in OS with regorafenib was maintained in all preplanned subgroup analyses. Median progression-free survival (mPFS) by modified RECIST was 3.1 months with regorafenib and 1.5 months with placebo. Median time to progression (mTTP) by modified RECIST was 3.2 vs. 1.5 months. Predefined subgroup analysis for mPFS and mTTP also showed consistent benefit. Adverse events (AEs) were reported in all regorafenib recipients (374 of 374, 100\%) and 179 of $193(93 \%)$ placebo recipients. The safety of regorafenib in HCC was consistent with the safety profile of regorafenib in other malignancies, and entailed no new safety concerns. The most common clinically relevant grade 3 or 4 treatment-emergent events were hypertension ( $15 \%$ in the regorafenib group versus $5 \%$ in the placebo group), hand-foot skin reaction (HFSR, $13 \%$ vs. 1\%), fatigue ( $9 \%$ vs. $5 \%$ ), and diarrhea (3\% vs. $0 \%$ ). Drug-related AEs that led to interruptions or dose reductions occurred with $202(54 \%)$ patients in the regorafenib group, and 20 (10\%) patients in the placebo group, while discontinuations occurred with $39(10 \%)$ patients in the regorafenib group and seven $(4 \%)$ patients in the placebo group. Of the 88 deaths reported during the study (50 patients in regorafenib group and 38 in placebo group), 7 cases were considered by the investigator to be related to the study drug in the regorafenib group and 2 cases in the placebo group, including 2 patients (1\%) with hepatic failure.

Interestingly, post-hoc exploratory analysis showed that HFSR with regorafenib was associated with improved mOS (14). Of the 379 patients who were randomized, 374 received at least 1 dose of regorafenib. Of the treated patients, $53 \%(\mathrm{n}=199)$ had HFSR of any grade and 13\% $(\mathrm{n}=47)$ had grade 3 HFSR. mOS was improved in patients who had HFSR at any time versus those who did not (14.1 vs. 6.6 months, HR $=0.52$, 95\% CI: $0.40-0.67$ ). Patients who had an HFSR event during cycle 1 also had 
more improved mOS versus those who did not (13.2 vs. 8.5 months, HR =0.66, 95\% CI: 0.51-0.86). However, subgroups of patients with and without HFSR at any time had some imbalances in their baseline characteristics. The potential confounding influence of baseline factors requires further investigation.

Recently, the additional analysis from the phase III RESORCE trial was reported (15). Median times from the start of sorafenib to death were 26.0 months (95\% CI: $22.6-$ 28.1 months) for regorafenib and 19.2 months (95\% CI: 16.3-22.8 months) for placebo. The sequence of sorafenib followed by regorafenib for HCC may extend survival beyond what has been previously reported.

The results of the RESORCE trial bring 10 years of negative randomized controlled trials (RCTs) in advanced HCC to an end. On April 27th, 2017, the U.S. Food and Drug Administration (FDA) formally approved the treatment of patients with HCC who have been previously treated with sorafenib as a new indication for regorafenib. It is only the second molecular targeted agent approved by the FDA for the treatment of HCC. This represents a major breakthrough and contribution in the treatment of HCC following sorafenib.

\section{Donafenib}

Donafenib is a new patent drug developed by Suzhou Zelgen Biopharmaceuticals with 3 deuterium methyl taking the place of a methyl on the molecule of sorafenib. As a deuterium compound, it has better metabolic stability and pharmacological effect. Donafenib has shown great potential in the treatment of different types of solid tumors including HCC by targeting Raf kinase and VEGFR tyrosine kinases. In vitro, donafenib can directly inhibit the proliferation of various tumor cells, including HCC, and has a very strong anti-angiogenesis effect. Bi et al. (16) reported a multicenter, phase 2 study of donafenib in patients with advanced HCC. In this randomized, open-label, parallel-group study, 106 patients with unresectable HCC, who had Child-Pugh class A liver function and received no prior systemic therapy were enrolled across 10 sites in China. All patients received oral donafenib 0.2 or $0.3 \mathrm{~g}$ BID for 4 weeks per cycle. mOS was 387 days in the $0.2 \mathrm{~g}$ group compared with 340 days in the $0.3 \mathrm{~g}$ group, and mTTP was 111 days in the $0.2 \mathrm{~g}$ group compared with 110 days in the 0.3 g group. At week 16, there were no complete responses according to RECIST 1.1 version in both groups, but partial response was confirmed in $2(5.0 \%)$ and $2(4.6 \%)$ patients in the $0.2 \mathrm{~g}$ group and the $0.3 \mathrm{~g}$ group, respectively; and stable disease was in $17(42.5 \%)$ and $18(40.9 \%)$ patients, in the $0.2 \mathrm{~g}$ group and the $0.3 \mathrm{~g}$ group, respectively.

The most common AEs, HFSR, liver dysfunction and leukopenia, were reported more frequently in the $0.3 \mathrm{~g}$ group. The median duration maintained for the initial dose in the two groups was 90 days in the $0.2 \mathrm{~g}$ group and 72 days in the $0.3 \mathrm{~g}$ group. Both regimens showed similar treatment responses for patients with HCC and $0.2 \mathrm{~g}$ BID seems to be appropriate.

A phase III trial (NCT02645981), evaluating the efficacy and safety of donafenib in patients with advanced HCC who had not received prior systemic therapy is ongoing. In total, 330 patients who have met the inclusion criteria have been randomly assigned to receive donafenib (0.2 $\mathrm{g}$ BID) or sorafenib (0.4 g BID). This is a non-inferiority trial and the primary endpoint is OS. The recruitment has ended and we are looking forward to the final result.

\section{Lenvatinib}

Similar to sorafenib, lenvatinib is also a novel tyrosine kinase inhibitor of multi-targets, including VEGFR, FGFR, PDGFR, RET and KIT. Two clinical trials have demonstrated that lenvatinib had a favorable safety and tolerability profile with evidence of antitumor activity on HCC $(17,18)$.

On Feb 9th, 2018, the result of the REFLECT research which aimed to compare the safety and effectiveness between lenvatinib and sorafenib for the treatment of advanced HCC patients with Child-Pugh class A, was published online in Lancet (19). This was a multicenter, randomized, open-label, phase III, non-inferiority trial that recruited patients with unresectable HCC, who had not received treatment for advanced disease, at 154 sites in 20 countries throughout the Asia-Pacific, European, and North American regions. Patients were randomly assigned $(1: 1)$ to receive oral lenvatinib $(\mathrm{n}=478,12 \mathrm{mg} / \mathrm{day}$ for bodyweight $\geq 60 \mathrm{~kg}$ or $8 \mathrm{mg} /$ day for bodyweight $<60 \mathrm{~kg}$ ) or sorafenib ( $\mathrm{n}=476) 400 \mathrm{mg}$ twice daily in 28 -day cycles. The primary endpoint was OS. The efficacy analysis followed the intention-to-treat principle, and only patients who received treatment were included in the safety analysis.

The findings demonstrated a median survival time of 13.6 months (95\% CI: 12.1-14.9 months) for lenvatinib which was non-inferior to sorafenib (12.3 months, HR $=0.92$, 95\% CI: 0.79-1.06), meeting criteria for noninferiority. Lenvatinib was also able to prolong mTTP (8.9 
vs. 3.7 months, $\mathrm{HR}=0.63$, 95\% CI: $0.53-0.73, \mathrm{P}<0.00001)$ and mPFS (7.4 vs. 3.8 months, $\mathrm{HR}=0.66,95 \% \mathrm{CI}$ : $0.57-0.77, \mathrm{P}<0.00001)$ in comparison with sorafenib. The objective response rate (ORR) by modified RECIST in the lenvatinib group was $24 \%$, which was significantly higher than the $9 \%$ rate in the sorafenib group $(\mathrm{P}<0.00001)$.

In terms of safety, both lenvatinib and sorafenib were consistent with previous reports. The most common anygrade AEs were hypertension (42\%), diarrhea (39\%), decreased appetite (34\%), and decreased weight (31\%) for lenvatinib, and palmar-plantar erythrodysesthesia (52\%), diarrhea (46\%), hypertension (30\%), and decreased appetite (27\%) for sorafenib. Median treatment duration was 5.7 months (range, 0 to 35.0 months) for lenvatinib and 3.7 months (range, 0.1 to 38.7 months) for sorafenib. Patients who received lenvatinib experienced fewer instances of palmar-plantar erythrodysesthesia, diarrhea, and alopecia, and more instances of hypertension, proteinuria, dysphonia, and hypothyroidism than did patients who received sorafenib. There were $13 \%$ of lenvatinib-treated and $9 \%$ of sorafenib-treated patients who had treatment discontinued due to AEs.

Especially noteworthy is that in this study (20), a total of 288 patients from China (including mainland China, Taiwan and Hong Kong) were enrolled, and the number of enrollment ranks the first among all countries or regions. Compared with the total population, the average age of the patients enrolled in China was relatively low, and a large number of patients with hepatitis $\mathrm{B}$ virus (HBV) were included (Chinese patients accounted for $83 \%$, and global accounted for $53 \%$ ). According to the subgroup analysis of Chinese patients, compared with sorafenib, lenvatinib reached its non-inferior criteria in the primary endpoint, namely, mOS, and mOS was significantly prolonged for 4.8 months (15.0 vs. 10.2 months, $\mathrm{HR}=0.73$, 95\% CI: $0.55-0.96, \mathrm{P}=0.026)$. At the same time, for patients with $\mathrm{HBV}$ infection, lenvatinib showed significant survival benefits (14.9 vs. 9.9 months, $\mathrm{HR}=0.72$, 95\% CI: $0.53-0.97$, $\mathrm{P}=0.026)$. Furthermore, for the secondary endpoints, lenvatinib was significantly better than sorafenib in mPFS (9.2 vs. 3.6 months, HR =0.55, 95\% CI: 0.42-0.72, $\mathrm{P}<0.00001)$, mTTP (11.0 vs. 3.7 months, HR $=0.53,95 \%$ CI: $0.40-0.71, \mathrm{P}<0.00001)$, and $\mathrm{RR}(21.5 \%$ vs. $8.3 \%$, $\mathrm{P}=0.00137)$. There was no significant difference between the Chinese subgroup and the total population in safety for lenvantinib and sorafenib.

In conclusion, lenvatinib was non-inferior to sorafenib in OS in untreated advanced HCC, and achieved statistically significant and clinically meaningful improvements in mPFS, mTTP, and ORR. For the Chinese patients, the effect was more significant, especially for patients with HBV. The safety profiles of lenvatinib and sorafenib in the study appear consistent with the known safety profiles of these drugs in HCC, and no new safety signals were identified. Based on these results, lenvatinib was formally approved for the treatment of unresectable HCC by the CFDA on September 5th, 2018. Ten years after sorafenib, lenvatinib is the second target drug approved for the first line treatment of HCC after, and thus signifies a crucial breakthrough in the systemic therapy of advanced HCC.

\section{Cabozantinib}

The FDA has already approved cabozantinib for the treatment of advanced medullary thyroid carcinoma and renal cell carcinoma. It is an oral agent which acts through the targeted inhibition of the MET, AXL and VEGFR 1-3 signaling pathways. While it is not a pure MET inhibitor, cabozantinib still has an obvious anti-angiogenesis effect on this pathway. Phase 2 data on cabozantinib in patients with advanced HCC who have been previously treated with sorafenib, are worthy of further study (21). CELESTIAL (NCT01908426) is a randomized, global phase 3 trial of cabozantinib versus placebo in this patient population. In October 2017, Exelixis Inc. announced that the CELESTIAL trial met its primary endpoint of OS, with cabozantinib providing a statistically significant and clinically meaningful improvement in mOS compared to placebo. Detailed results were reported at the ASCO 2018 Gastrointestinal Cancers Symposium and Annual Meeting (22). In the study, a total of 707 patients with advanced previously-treated HCC (sorafenib and/or other systemic therapy) were randomized at a ratio of $2: 1$ to receive cabozantinib (60 $\mathrm{mg} \mathrm{qd}$ ) or placebo, stratified by disease etiology (HBV, HCV, other), geographic region (Asia, other), and presence of extrahepatic spread and/ or macrovascular invasion. Baseline characteristics were balanced between the two arms. After 484 deaths had occurred (317 out of 470 for cabozantinib; 167 out of 237 for placebo), the study met the primary endpoint at the second planned interim analysis with $\mathrm{mOS}$ at 10.2 months for cabozantinib versus 8.0 months for placebo $(\mathrm{HR}=0.76,95 \% \mathrm{CI}: 0.63-0.92, \mathrm{P}=0.0049)$. Median PFS was 5.2 vs. 1.9 months $(\mathrm{HR}=0.44,95 \% \mathrm{CI}: 0.36-0.52$, $\mathrm{P}<0.001)$, and ORR was $4 \%$ versus $0.4 \%(\mathrm{P}=0.0086)$. The safety data in the study were consistent with the established 
profile of cabozantinib. The most common grade $3 / 4$ AEs (predominantly grade 3) with higher incidence in the cabozantinib arm included HFSR (17\%), hypertension $(16 \%)$, increased aspartate aminotransferase (12\%), fatigue $(10 \%)$, and diarrhea $(10 \%)$. The outcomes of patients who received only prior sorafenib were also analyzed based on duration of prior therapy (23). Out of the 495 patients who received only prior sorafenib, $136(27 \%)$ received prior sorafenib for 3 months, 141 (28\%) for 3 to 6 months, and 217 (44\%) for above 6 months. For these patients, mOS was 11.3 months for cabozantinib versus 7.2 months for placebo $(\mathrm{HR}=0.70)$, and $\mathrm{mPFS}$ was 5.5 versus 1.9 months $(\mathrm{HR}=0.40)$. Grade $3 / 4 \mathrm{AEs}$ in the subgroups were similar to those observed in the overall population in both arms. Cabozantinib improved OS and PFS in patients with advanced HCC who had received sorafenib as the only prior systemic therapy irrespective of the duration of prior sorafenib treatment. Another subgroup analysis (24) of the trial based on age ( $<65$ and $\geq 65$ years) showed that cabozantinib improved OS and PFS versus placebo irrespective of age category. The discontinuation rate due to treatment-related AEs in the cabozantinib group was lower in patients $<65$ versus $\geq 65$ years old $(11 \%$ vs. $22 \%$ ), while the percentage of patients with any dose reduction $(61 \%$ vs. $64 \%)$ and the median average daily dose of cabozantinib (37 vs. $34 \mathrm{mg}$ ) were similar in both age groups. The most common grade $3 / 4$ AEs in both age groups were consistent with those in the overall population.

Based on these results, Exelixis has submitted a supplemental New Drug Application for cabozantinib as a treatment for patients with previously treated advanced HCC to the FDA. The FDA has completed its filing review and determined that the application is sufficiently complete to permit a substantive review. The filing has been assigned a Prescription Drug User Fee Act action date of January 14th, 2019.

\section{Ramucirumab}

Ramucirumab is a recombinant IgG1 monoclonal antagonist antibody against VEGF receptor-2. The FDA has already approved ramucirumab in combination with chemotherapy for the treatment of metastatic colorectal cancer, non-small cell lung cancer, gastric cancer and adenocarcinoma of the gastroesophageal junction.

Unfortunately, the result of the REACH trial (8), a randomized, placebo-controlled, double-blind, multicenter, phase 3 trial (NCT 01140347), showed that second-line treatment with ramucirumab did not significantly improve survival over placebo in patients with advanced HCC. mOS for the ramucirumab group was 9.2 months (95\% CI: 8.0-10.6) vs. 7.6 months (95\% CI: 6.0-9.3) for the placebo group (HR $=0.87,95 \% \mathrm{CI}: 0.72-1.05, \mathrm{P}=0.14)$. However, researchers found that in patients with baseline AFP levels of $400 \mathrm{ug} / \mathrm{L}$ or more, a ramucirumab survival benefit was observed for Child-Pugh scores of 5 ( $\mathrm{HR}=0.61,95 \% \mathrm{CI}$ : $0.43-0.87, \mathrm{P}=0.01)$ and 6 (HR $=0.64,95 \% \mathrm{CI}: 0.42-0.98$, $\mathrm{P}=0.04)(25)$.

In order to further verify this finding, a new phase III trial REACH-2 (NCT02435433) was designed. The patients enrolled in this trial were required to have AFP $\geq 400 \mathrm{ng} / \mathrm{mL}$ at baseline. The primary endpoint of the REACH-2 trial was OS and key secondary endpoints included PFS, ORR, quality of life, and safety. Detailed results of the trial were just reported on at the ASCO 2018 Annual Meeting (26). A total of 292 patients across 20 countries in North America, Asia, Europe and Latin America, were randomized to the ramucirumab arm or placebo arm. Baseline characteristics were well balanced between the 2 arms. Ramucirumab treatment significantly improved OS (mOS 8.5 vs. 7.3 months, $\mathrm{P}=0.0199$ ), and $\mathrm{mPFS}$ was also significantly improved (2.8 vs. 1.6 months, $\mathrm{P}=0.0001$ ). Grade $3 \mathrm{AEs}$, which occurred in $5 \%$ patients in the ramucirumab arm, included hypertension (12.2\%) and hyponatremia (5.6\%). The REACH-2 trial met its primary endpoint showing a significant survival benefit for ramucirumab treatment by reducing the risk of death (29\%) in patients with $\mathrm{HCC}$ and $\mathrm{AFP} \geq 400 \mathrm{ng} / \mathrm{mL}$ who progressed on or were intolerant to sorafenib, as well as the secondary endpoint of PFS. Treatment was also well-tolerated, with a safety profile consistent with the established profile for single-agent ramucirumab.

REACH-2 is the first positive study in a biomarker selected patient population which demonstrates a significant and meaningful OS benefit and safety profile in HCC patients with baseline AFP $\geq 400 \mathrm{ug} / \mathrm{L}$, a population typically associated with poor prognosis. As such, it promises to be a new option in the second-line treatment of advanced HCC.

\section{Apatinib}

Apatinib is a novel oral multi-kinase inhibitor of the VEGF receptor-2. In a preclinical study, Yang et al. (27) found that the concentration-dependent anti-proliferative effects of apatinib were observed in $6 \mathrm{HCC}$ cell lines tested in vitro, and response to apatinib was significantly correlated with 
VEGFR-2 expression level. Moreover, apatinib significantly inhibited the VEGF-triggered VEGFR-2 phosphorylation and activation of downstream signaling molecules such as AKT and ERK1/2. Apatinib could also induce a cell-cycle arrest at the G2/M phase and promote HCC apoptosis. In vivo data showed that apatinib can effectively inhibit tumor growth associated with increased HCC cell apoptosis, decreased angiogenesis, and prolonged survival in a mouse xenograft model of human HCC. These findings provide a rationale for adopting clinical trials of apatinib as a single agent or in combination with other agents in patients with HCC.

Qin et al. (28) reported a multicenter, randomized, openlabel, dose-finding, phase II clinical study of apatinib as a first-line treatment in Chinese patients with advanced HCC (NCT01192971). A total of 121 treatment naive patients with advanced HCC and Child-Pugh liver function class A were randomized to receive apatinib 850 or $750 \mathrm{mg} /$ day. The primary endpoint of the trial was mTTP. Secondary endpoints included OS, ORR and disease control rate (DCR). The baseline characteristics of the 2 groups were similar with regards to ECOG scores, number of metastatic sites, pathological grading and prior therapy $(\mathrm{P}>0.05)$. mTTPs of the $850 \mathrm{mg}$ group and the $750 \mathrm{mg}$ group were 4.2 and 3.3 months respectively. The mOS and DCR of the 2 groups were 9.7 vs. 9.8 months, and $48.57 \%$ vs. $37.25 \%$. Apatinib was well-tolerated. Most of the AEs could be managed by dose interruptions or reductions. There was no significant favorable safety profile between the 2 groups.

A multicenter, randomized, double-blind, phase III trial (NCT02329860), evaluating the efficacy and safety of apatinib in patients with advanced liver cancer who have progressed on systemic therapy (chemotherapy and/or targeted therapy) is ongoing. More than 400 patients who met the entry criteria have been randomly assigned in a 2:1 ratio to receive apatinib or placebo. The primary endpoint of the study is OS. At present, the enrollment has ended. The final result is much anticipated.

\section{Anti-angiogenesis combined with immune checkpoint inhibitors}

In recent years, with the new findings on tumor immune escape and immune tolerance mechanisms, tumor immunotherapy has developed rapidly. Specifically, immune checkpoint inhibitors, such as ipilimumab (anti CTLA-4 antibody), atezolizumab (anti-PD-L1 monoclonal antibody), pembrolizumab and nivolumab (anti-PD-1 monoclonal antibody), have proven successful in the treatment of many tumors. Based on the tumor response rate and durability of response from the phase 1/2 CheckMate 040 trial (29), the FDA has approved nivolumab for the treatment of patients with HCC following prior sorafenib, regardless of PD-L1 status. A new era of immunotherapy for HCC has begun.

Theoretically, anti-angiogenesis therapy can normalize tumor vessels, thus changing the immunosuppressive environment of HCC and allowing more therapeutic drugs to absorb into tumor tissue. Consequently, combination therapy with immune checkpoint inhibitors and antiangiogenesis agents may have a synergistic effect. This notion has received increasing attention in recent years.

A nonclinical study demonstrated that lenvatinib could enhance antitumor activity of a PD-1 blockade agent by potentiating Th1 immune response (30). In patients with endometrial cancers, combination therapy with pembrolizumab and lenvatinib showed high response rates (50-70\%) and long-lasting durable response (31). A phase Ib trial (32) of lenvatinib plus pembrolizumab (NCT03006926) in HCC is currently being conducted in Japan, testing the efficacy of this combination. As of December 1st, 2017, 18 patients had received lenvatinib plus pembrolizumab therapy. The most common AEs were decreased appetite and hypertension (56\% each). No new safety signals were identified. At data cutoff, tumor reduction baseline was observed in all evaluable patients except one. Preliminary results showed the combination was well-tolerated with encouraging anti-tumor activity in patients with unresectable HCC.

Atezolizumab plus bevacizumab is another combination. A phase III multicenter, randomized, open-label, IMmotion 151 study (33), has shown improvement in investigator-assessed PFS compared with sunitinib alone (11.2 vs. $7.7, \mathrm{HR}=0.74 ; 95 \% \mathrm{CI}: 0.57-0.96 ; \mathrm{P}=0.02$ ) for patients with inoperable, locally advanced or metastatic renal cell carcinoma who have not received prior systemic treatment and whose disease expressed PD-L1. For HCC, Stein et al. (34) at the recent ASCO 2018 Annual Meeting, reported the results of a phase Ib study evaluating the safety and clinical activity of atezolizumab plus bevacizumab in HCC patients who were naive to systemic therapy. Patients received atezolizumab $(1,200 \mathrm{mg})$ plus bevacizumab $(15 \mathrm{mg} / \mathrm{kg})$ IV every 3 weeks until loss of clinical benefit or unacceptable toxicity. As of the data cutoff (October 24th, 2017), 26 patients were evaluable for safety. Treatmentrelated all-grade AEs occurred in 21 patients (81\%) and grade 3-4 AEs were seen in 9 patients (35\%), most 
commonly hypertension $(\mathrm{n}=5)$. No grade 5 AEs were observed. Immune-related AEs requiring corticosteroid therapy occurred in 3 patients (12\%). Among 21 efficacyevaluable patients, confirmed partial responses occurred in 13 (62\%). At the ESMO 2018 Annual Meeting in October, the data were updated further (35). At clinical data cutoff (July 26th, 2018), 103 patients with HCC treated with atezolizumab plus bevacizumab were evaluable for safety and 73 patients were evaluable for efficacy with a minimum follow-up of 16 weeks. According to the central independent imaging evaluation, there were 4 cases of CR, 16 cases of PR, and ORR was $27 \%$. The mPFS was 14.9 months and mOS has not been reached. The combination of atezolizumab plus bevacizumab is safe and well-tolerated, and no new safety signals were identified beyond the reported safety profile for each agent. The confirmed response rate of $62 \%$ suggests the combination has synergistic clinical activity. A phase III, open-label, randomized study $(36,37)$ of atezolizumab in combination with bevacizumab compared with sorafenib in patients with untreated locally advanced or metastatic HCC (YO40245/ NCT03434379) is currently recruiting patients. Patients will be randomized 2:1 to receive atezolizumab $(1,200 \mathrm{mg})$ plus bevacizumab $(15 \mathrm{mg} / \mathrm{kg}$ ) IV Q3W or sorafenib (400 mg PO BID) until loss of clinical benefit or unacceptable toxicity. Approximately 480 patients will be enrolled globally.

In addition to this, there are several ongoing clinical trials of combination treatments with immune checkpoint inhibitors and anti-angiogenesis agents, such as durvalumab plus ramucirumab (NCT02572687), nivolumab plus cabozantinib (NCT01658878), PDR001 (an antiPD-1 monoclonal antibody developed by Novartis) plus sorafenib (NCT02988440), etc. SHR1210 is a Chinese domestic humanized monoclonal antibody against PD-1. In a multicenter phase 2 study (38) to evaluate SHR-1210 in Chinese subjects with advanced HCC who failed or were intolerant to prior systemic treatment, a total of 220 patients were included. In the 217 patients evaluable for efficacy, ORR was $13.8 \%$ and survival rate at 6 months was $74.7 \%$. mTTP was 2.7 months and mPFS was 2.1 months. mOS has not been reached but it was estimated to be more than 14.4 months.

SHR1210 plus apatinib in a phase 1 trial (39) demonstrated manageable toxicity in HCC, gastric cancer or esophagogastric junction cancer patients. Particularly encouraging clinical activity (ORR and DCR were 50.0\% and $85.7 \%$, respectively in 14 evaluable patients) was observed in patients with pretreated, advanced HCC. A phase 2 trial of SHR-1210 in combination with apatinib in patients with advanced HCC (NCT03463876) is ongoing as well. Preliminary results have shown that the possible optimal dose for combination is apatinib $375 \mathrm{mg} /$ day po qd and SHR$12102 \mathrm{mg} / \mathrm{kg}$ iv.gtt q2w.

\section{Summary}

HCC is one of the most common malignancies with high morbidity and mortality in the world, especially in China. It is very difficult to treat advanced HCC.

Since a new field of anti-angiogenesis therapy for cancer has been opened, more and more drugs have emerged. Anti-angiogenesis therapy has played an increasingly important role in the treatment of various cancers, including HCC. As one of these anti-angiogenesis drugs, sorafenib has been a breakthrough for the dilemma facing the treatment of advanced HCC. Sorafenib has been approved in advanced HCC for 11 years. In the past decade, although we have experienced many research failures, we have also gained invaluable experience thereby. Since 2017, there has been a surge of promising news in the systemic therapy of advanced HCC. The FDA has approved the new indication of regorafenib and nivolumab for the treatment of patients with HCC who have been previously treated with sorafenib. Three phase 3 trials, REFLECT for lenvatinib, CELESTIAL for cabozantinib and REACH-2 for ramucirumab, have achieved statistical significance and clinically meaningful outcomes, and are pending approval by the FDA. In addition, the eagerly anticipated results of the donafenib and apatinib trials are just on the horizon. As can be seen, the systemic treatment of advanced HCC has made encouraging progress and entered a new era. Anti-angiogenesis therapy has proven to be pivotal to the comprehensive treatment of HCC.

Nonetheless, there are many questions which remain to be solved in the future: how to combine anti-angiogenesis therapy with other means, such as TACE, systemic chemotherapy and immune targeted therapies, in order to improve the efficacy further? How to screen the "right patient" through clinical characteristics and/or molecular markers that can predict the efficacy? How to deal with the adverse reactions of anti-angiogenesis drugs, especially certain toxicities, in order to improve tolerance and quality of life? All these challenges are the focus of future clinical research. We have good reason to believe that with the expanding depth of studies, with the increase in the number 
of promising drugs, and with the improvement of treatment effect, one day, HCC will no longer reign as "the king of cancer".

\section{Acknowledgements}

None.

\section{Footnote}

Conflicts of Interest: The authors have no conflicts of interest to declare.

\section{References}

1. Bray F, Ferlay J, Soerjomataram I, et al. Global Cancer Statistics 2018: GLOBOCAN Estimates of Incidence and Mortality Worldwide for 36 Cancers in 185 Countries. CA Cancer J Clin 2018. [Epub ahead of print].

2. Chen WQ, Li H, Sun KX, et al. Report of Cancer Incidence and Mortality in China, 2014. Zhonghua Zhong Liu Za Zhi 2018;40:5-13.

3. Global Burden of Disease Liver Cancer Collaboration, Akinyemiju T, Abera S, et al. The Burden of Primary Liver Cancer and Underlying Etiologies From 1990 to 2015 at the Global, Regional, and National Level Results From the Global Burden of Disease Study 2015. JAMA Oncol 2017;3:1683-91.

4. Johnson PJ, Qin S, Park JW, et al. Brivanib versus sorafenib as first-line therapy in patients with unresectable, advanced hepatocellular carcinoma: results from the randomized phase III BRISK-FL study. J Clin Oncol 2013;31:3517-24.

5. Llovet JM, Decaens T, Raoul JL, et al. Brivanib in patients with advanced hepatocellular carcinoma who were intolerant to sorafenib or for whom sorafenib failed: results from the randomized phase III BRISK-PS study. J Clin Oncol 2013;31:3509-16.

6. Cheng AL, Kang YK, Lin DY, et al. Sunitinib versus sorafenib in advanced hepatocellular cancer: results of a randomized phase III trial. J Clin Oncol 2013;31:4067-75.

7. Cainap C, Qin S, Huang WT, et al. Linifanib Versus Sorafenib in Patients With Advanced Hepatocellular Carcinoma: Results of a Randomized Phase III Trial. J Clin Oncol 2015;33:172-9.

8. Zhu AX, Park JO, Ryoo BY, et al. Ramucirumab versus placebo as second-line treatment in patients with advanced hepatocellular carcinoma following first-line therapy with sorafenib (REACH): a randomised, double-blind, multicentre, phase 3 trial. Lancet Oncol 2015;16:859-70.

9. Zhu AX, Kudo M, Assenat E, et al. Effect of everolimus on survival in advanced hepatocellular carcinoma after failure of sorafenib in the EVOLVE-1 randomized clinical trial. JAMA 2014;312:57-67.

10. Rimassa L, Assenat E, Peck-Radosavljevic M, et al. Second-line tivantinib (ARQ 197) vs placebo in patients (Pts) with MET-high hepatocellular carcinoma (HCC): results of the METIV-HCC phase III trial. J Clin Oncol 2017;35:4000.

11. Kang YK, Yau T, Park JW, et al. Randomized phase II study of axitinib versus placebo plus best supportive care in second-line treatment of advanced hepatocellular carcinoma. Ann Oncol 2015;26:2457-63

12. Bruix J, Tak WY, Gasbarrini A, et al. Regorafenib as secondline therapy for intermediate or advanced hepatocellular carcinoma: multicentre, open-label, phase II safety study. Eur J Cancer 2013;49:3412-9.

13. Bruix J, Qin S, Merle P, et al. Regorafenib for patients with hepatocellular carcinoma who progressed on sorafenib treatment (RESORCE): a randomised, double-blind, placebo-controlled, phase 3 trial. Lancet 2017;389:56-66.

14. Bruix J, Merle P, Granito A, et al. Hand-foot skin reaction (HFSR) and overall survival (OS) in the phase 3 RESORCE trial of regorafenib for treatment of hepatocellular carcinoma (HCC) progressing on sorafenib. J Clin Oncol 2018;36:412.

15. Finn RS, Merle P, Granito A, et al. Outcomes of sequential treatment with sorafenib followed by regorafenib for HCC: Additional analyses from the phase III RESORCE trial. J Hepatol 2018;69:353-8.

16. Bi F, Qiu M, Chai X, et al. A Multicenter Phase II Study of Donafenib in Patients with Advanced Hepatocellular Carcinoma. J Clin Oncol 2017;35 suppl: abstract 188934.

17. Koyama N, Saito K, Nishioka Y, et al. Pharmacodynamic change in plasma angiogenic proteins: a dose-escalation phase 1 study of the multi-kinase inhibitor lenvatinib. BMC Cancer 2014;14:530.

18. Ikeda M, Okusaka T, Mitsunaga S, et al. Safety and pharmacokinetics of lenvatinib in patients with advanced hepatocellular carcinoma. Clin Cancer Res 2016;22:1385-94.

19. Kudo M, Finn RS, Qin S, et al. Lenvatinib versus sorafenib in first-line treatment of patients with unresectable hepatocellular carcinoma: a randomised phase 3 noninferiority trial. Lancet 2018;391:1163-73

20. Qin SK. Lenvatinib versus sorafenib in first-line treatment of patients with unresectable hepatocellular carcinoma: 
Subgroup analysis of Chinese patients. Presentation on 2017 Chinese Society of Clinical Oncology Annual Meeting.

21. Verslype C, Cohn AL, Kelley RK, et al. Activity of cabozantinib (XL184) in hepatocellular carcinoma patients (pts): results from a Phase II randomized discontinuation trial (RDT). J Clin Oncol 2012;30:261.

22. Ghassan K. Abou-Alfa, Tim Meyer, et al. Cabozantinib (C) versus placebo $(\mathrm{P})$ in patients (pts) with advanced hepatocellular carcinoma (HCC) who have received prior sorafenib: Results from the randomized phase III CELESTIAL trial. J Clin Oncol 2018;36:207.

23. Kelley RK, Ryoo BY, Merle P, et al. Outcomes in patients (pts) who had received sorafenib (S) as the only prior systemic therapy in the phase 3 CELESTIAL trial of cabozantinib $(\mathrm{C})$ versus placebo $(\mathrm{P})$ in advanced hepatocellular carcinoma (HCC). J Clin Oncol 2018;36:4088.

24. Rimassa L, Cicin I, Blanc JF, et al. Outcomes based on age in the phase 3 CELESTIAL trial of cabozantinib (C) versus placebo $(\mathrm{P})$ in patients (pts) with advanced hepatocellular carcinoma (HCC). J Clin Oncol 2018;36:4090.

25. Zhu AX, Baron AD, Malfertheiner P, et al. Ramucirumab as Second-Line Treatment in Patients With Advanced Hepatocellular Carcinoma: Analysis of REACH Trial Results by Child-Pugh Score. JAMA Oncol 2016;3:235-43.

26. Zhu AX, Kang YK, Yen CJ, et al. A randomized, doubleblind, placebo-controlled phase 3 study of ramucirumab versus placebo as second-line treatment in patients with advanced hepatocellular carcinoma (HCC) and elevated baseline alpha-fetoprotein (AFP) following first-line sorafenib. J Clin Oncol 2018;36:4003.

27. Yang CX, Qin SK. Study on the inhibitory effects of apanitinib on HCC cell lines and its correlated mechanisms. Post-doctoral thesis of Post-doctoral scientific research station in Nanjing General Hospital of PLA Eastern Theater, 2014.

28. Qin SK. Apatinib in Chinese patients with advanced hepatocellular carcinoma: A phase II randomized, openlabel trial. J Clin Oncol 2014;32:4019.

29. El-Khoueiry AB, Sangro B, Yau T, et al. Nivolumab in patients with advanced hepatocellular carcinoma (CheckMate 040): an open-label, non-comparative, phase $1 / 2$ dose escalation and expansion trial. Lancet 2017;389:2492-502.

30. Kato T, Bao X, Macgrath S, et al. Lenvatinib mesilate (LEN) enhanced antitumor activity of a PD-1 blockade agent by potentiating Th1 immune response. Ann Oncol 2016;27:2PD.

31. Makker V, Rasco DW, Dutcus CE, et al. A phase Ib/II trial of lenvatinib (LEN) plus pembrolizumab (Pembro) in patients (Pts) with endometrial carcinoma. J Clin Oncol 2017;35:5598.

32. Ikeda M, Sung MW, Kudo M, et al. A phase $1 \mathrm{~b}$ trial of lenvatinib (LEN) plus pembrolizumab (PEM) in patients (pts) with unresectable hepatocellular carcinoma (uHCC). J Clin Oncol 2018;36: abstract 4076.

33. Motzer RJ, Powles T, Atkins MB, et al. IMmotion151: A Randomized Phase III Study of Atezolizumab Plus Bevacizumab vs Sunitinib in Untreated Metastatic Renal Cell Carcinoma (mRCC). J Clin Oncol 2018;36:578.

34. Stein S, Pishvaian MJ, Lee MS, et al. Safety and clinical activity of $1 \mathrm{~L}$ atezolizumab + bevacizumab in a phase $\mathrm{Ib}$ study in hepatocellular carcinoma (HCC). J Clin Oncol 2018;36:4074.

35. Pishvaian MJ, Lee MS, Ryoo BY, et al. Phase Ib study of atezolizumab + bevacizumab in HCC-update on safety and clinical activity. Ann Oncol 2018;29:LBA26.

36. Finn RS, Ducreux M, Qin S, et al. IMbrave150: A randomized phase III study of $1 \mathrm{~L}$ atezolizumab plus bevacizumab vs sorafenib in locally advanced or metastatic hepatocellular carcinoma. J Clin Oncol 2018;36:abstract TPS4141.

37. Ducreux MP, Cheng A, Qin S, et al. Atezolizumab + bevacizumab vs sorafenib in locally advanced or metastatic hepatocellular carcinoma: The randomised phase III study IMbrave150. Ann Oncol 2018;29:viii205-70.

38. Qin S, Ren Z, Meng Z, et al. A Randomized Multicenter Phase 2 Study to Evaluate SHR-1210 (PD-1 Antibody) in Subjects with Advanced Hepatocellular Carcinoma (HCC) who Failed or Were Intolerant to Prior Systemic Treatment. Ann Oncol 2018;29:LBA27.

39. Xu JM, Zhang Y, Jia R, et al. Anti-programmed death-1 antibody SHR-1210 (S) combined with apatinib (A) for advanced hepatocellular carcinoma (HCC), gastric cancer (GC) or esophagogastric junction (EGJ) cancer refractory to standard therapy: A phase 1 trial. J Clin Oncol 2018;36:4075.

Cite this article as: Gong X, Qin S. Study progression of anti-angiogenetic therapy and its combination with other agents for the treatment of advanced hepatocellular carcinoma. HepatoBiliary Surg Nutr 2018;7(6):466-474. doi: 10.21037/ hbsn.2018.11.04 